\title{
Diacronie
}

Studi di Storia Contemporanea

$\mathrm{N}^{\circ}$ 9, 1 | 2012

Quando la classe operaia andava in paradiso

\section{Alexander Höbel, Il PCI di Luigi Longo (1964-1969)}

\section{Marco Albeltaro}

\section{(2) OpenEdition}

\section{Journals}

\section{Edizione digitale}

URL: http://journals.openedition.org/diacronie/3107

DOI: 10.4000/diacronie.3107

ISSN: 2038-0925

\section{Editore}

Association culturelle Diacronie

\section{Notizia bibliografica digitale}

Marco Albeltaro, « Alexander Höbel, I/ PCl di Luigi Longo (1964-1969) », Diacronie [Online], N 9, 1 | 2012 documento 18, Messo online il 29 janvier 2012, consultato il 24 septembre 2020. URL : http:// journals.openedition.org/diacronie/3107; DOI : https://doi.org/10.4000/diacronie.3107 


\section{Diacronie}

N. 9 | 1|2012 Quando la classe operaia andava in paradiso

18/

\section{RECENSIONE:}

\section{Alexander, HÖBEL, Il PCI di Luigi Longo (1964-1969), Napoli, Edizioni Scientifiche Italiane, 2010, 626 pp.}

A cura di Marco ALBELTARO *

\section{Luigi Longo: da traghettatore a protagonista1}

Il libro di Alexander Höbel - Il PCI di Luigi Longo (1964-1969), prefazione di Francesco Barbagallo, Napoli, Edizioni Scientifiche Italiane, 2010, 626 pp.) è e rimarrà a lungo un contributo fondamentale alla storia del Partito comunista italiano e alla biografia di Luigi Longo.

Volume ricchissimo di documenti, copre un arco cronologico ristretto: il quinquennio in cui Luigi Longo è stato Segretario del PCI, imprimendo al più grande partito comunista occidentale il segno della sua politica. Schiacciato fra Togliatti da un lato e Berlinguer dall'altro (che diviene vice-segretario nel 1969, ipotecando in qualche modo la futura leadership del partito), il periodo di direzione del comunista piemontese è stato a lungo stato considerato come una semplice fase di transizione.

La storiografia più recente ci ha mostrato come in realtà la segreteria di Longo abbia avuto una sua specificità ed una sua importanza ${ }^{2}$. Proprio da questa considerazione muove l'analisi storiografica di Höbel. Un'analisi che si fonda su una mole impressionante di documenti, frutto di anni di ricerche presso la Fondazione Gramsci, l'Archivio Centrale dello Stato e molte altre sedi archivistiche, su una conoscenza dettagliatissima della storiografia italiana e internazionale con la quale l'autore riesce a dialogare rendendo pienamente la complessità dell'analisi storica.

${ }^{1}$ Una prima versione di questa recensione è apparsa nel numero 5 del 2011 di «Marx ventuno». 2 Cfr. almeno GUERRA, Adriano, La solitudine di Berlinguer. Governo, etica e politica dal "no" a Mosca alla questione morale, Roma, Ediesse, 2007; SORGONÀ, Gregorio, La svolta incompiuta. Il gruppo dirigente del PCI dall'VIII all'XI congresso (1956-1965), Roma, Aracne, 2011. 
Ci sono alcuni snodi fondamentali nel libro di Höbel su cui è utile soffermarsi in quanto costituiscono l'ossatura della scelta interpretativa dell'autore. Una scelta interpretativa che coraggiosamente - dati i tempi si potrebbe anche dire temerariamente! - e orgogliosamente, non nasconde la simpatia per l'oggetto di studio. Nelle pagine di Höbel appare infatti la tensione dell'autore nel cercare nella storia che racconta degli elementi di attualità, degli spunti di riflessione per il presente, talvolta calcando un po' la mano, ma senza permettere mai alla passione politica di prendere il sopravvento sul rigore scientifico. Lo stesso confronto con la storiografia mostra una notevole capacità di mettere fra loro in connessione le interpretazioni che si sono stratificate in decenni, discutendole, criticandole talvolta, ma sempre all'insegna di un fruttuoso dibattito intellettuale.

Höbel nell'analizzare la specificità della segreteria di Longo passa in rassegna con particolare attenzione tutte quelle fasi che hanno segnato il quinquennio 1964-1969. Dalla coraggiosa decisione di pubblicare il memoriale scritto da Togliatti a Yalta poco prima di morire, nonostante la netta opposizione dei sovietici, all'apertura verso i movimenti del Sessantotto, dando vita ad un dialogo non certo facile con gli studenti, fino all'opposizione contro l'intervento delle truppe del patto di Varsavia in Cecoslovacchia, il tutto all'insegna di una nuova stagione di democrazia interna nel partito. ̀̀ infatti Longo il segretario comunista che più attribuisce alla Direzione un ruolo di primaria importanza nell'elaborazione della linea politica.

Uno dei punti su cui Höbel si concentra maggiormente è il dialogo aperto da Longo col movimento studentesco. L'autore rigetta da subito la vulgata che vedrebbe nel PCI un ritardo nella comprensione del significato del Sessantotto. Sullo sfondo delle dinamiche politiche interne al partito, l'autore inserisce un tema cruciale: la differenza che esiste fra due orizzonti che finiscono per intrecciarsi ma anche per scontrarsi. Da un lato vi è infatti una cultura politica novecentesca, quella del PCI e dei suoi dirigenti, legata a categorie che spesso il movimento studentesco contesta; dall'altro c'è il movimento, con tutto il suo carico di energie contestatarie che affondano le proprie radici in un terreno politico nuovo, che a tratti sembra rapito da istanze post-moderne, che infatti non possono essere fatte proprie da un partito comunista novecentesco. In questo quadro lo sforzo di Longo, così come descritto da Höbel, appare in tutta la sua importanza e originalità: si tratta di un percorso politico che nasce innanzitutto dalla voglia di capire e di interpretare la novità - seppur filtrandola - sia attraverso una cultura politica storicamente radicata (quella del PCI), sia in rapporto alle differenti anime del partito, che vanno sempre più componendosi in un patchwork generazionale. 
Il tema del metodo di direzione politica è un altro degli elementi che contribuiscono a restituire importanza alla segreteria di Longo. Se la figura carismatica di Togliatti aveva in qualche modo avocato a sé l'onere della sintesi del dibattito politico interno come avrebbe del resto fatto l'altro segretario carismatico per eccellenza, Enrico Berlinguer - Luigi Longo, conscio della delicatezza del suo ruolo dopo la leadership incontrastata di Togliatti, restituisce alla Direzione del partito un posto di primo piano. Nello studio di Höbel questo elemento appare in modo molto chiaro anche per la grandissima attenzione che l'autore mette nel seguire passo dopo passo tutti i dibattiti degli organismi dirigenti: un'attenzione talvolta perfino eccessiva, ma che ha il merito di battere ogni possibile pista di ricerca.

L'attenzione di Longo verso il dibattito interno al PCI si riflette anche in un'altrettanto sincera considerazione del ruolo degli organismi parlamentari. È proprio su questo terreno che il PCI di Longo costruisce un altro dei fattori della sua specificità. Il rapporto fra il PCI e i governi Moro, ad esempio, è una delle questioni che Höbel indaga con maggiore originalità. L'autore affronta con decisione il tema delle presunte responsabilità del PCI nel fallimento dei governi di centro-sinistra, riuscendo a smontare una vulgata fondata più sul pregiudizio politico che sul riscontro della verità storica. Le ragioni del fallimento di quell'esperienza politica non vanno infatti attribuite ad una sorta di pregiudizio ideologico di parte comunista: anzi, il PCI mostra in quella fase un atteggiamento pragmatico e una notevole flessibilità sia sui grandi temi - primo fra tutti il dialogo coi cattolici sul problema della pace -, sia su singoli problemi. La capacità mostrata dal PCI di Longo di incalzare i governi con proposte parlamentari sempre supportate da un'azione di massa nel paese portò infatti a risultati concreti sul piano legislativo.

Höbel mostra, insomma, tutto il senso di responsabilità politica e civile di cui Longo fu capace, nel continuo sforzo di tenere unito il partito nelle sue componenti interne. Proprio riguardo l'atteggiamento verso il centro-sinistra si aprì infatti nei gruppi dirigenti del PCI un dibattito molto vivace, una vera e propria «esplosione» della dialettica interna, come la definisce Höbel. Il libro ne traccia un'articolata geografia fin dalla proposta di Amendola del «partito unico» - un fulmine a ciel sereno - passando per le suggestioni di Pietro Ingrao, per approdare ancora una volta al ruolo arbitrale di Longo. Proprio prendendo le mosse da questi dibattiti, Höbel riposiziona nella corretta griglia interpretativa l'XI congresso che, con buona pace di una parte della storiografia 
e della memorialistica ${ }^{3}$, non assume affatto i tratti della «resa dei conti» fra la destra e la sinistra interne al PCI; è piuttosto un'assise cruciale che riesce, pur non senza scontri aspri, a introiettare nel programma politico del partito alcune delle istanze proposte proprio dalla sinistra, come mostrano le Tesi conclusive, studiate da Höbel con molta attenzione.

Un altro tema cui l'autore dedica pagine importanti è quello del ruolo internazionale di Longo. Höbel mostra bene come il segretario comunista col trascorrere del tempo sia divenuto una delle figure più importanti del movimento comunista internazionale mostrando, fin dai primi incontri di Yalta, la capacità di rapportarsi ai sovietici senza complessi di inferiorità: si pensi all'appoggio dato da Longo alla primavera di Praga e al conseguente dissenso coi sovietici che aprì una strada che poi sarebbe stata percorsa, in modo plateale, da Enrico Berlinguer. La corposa mole di documentazione che Höbel analizza riguardo a questa fase mette in luce come Longo, in politica internazionale, sia stato il vero precursore di un atteggiamento che avrebbe poi caratterizzato il PCI negli anni successivi.

La ricerca di Höbel, una ricerca di «lungo periodo» come precisa l'autore stesso, è innanzitutto uno studio di storia politica, perfino troppo politica. La grandissima attenzione ai dibattiti interni al partito, a quelli parlamentari, alle vicende degli altri attori della politica italiana, finisce infatti per lasciare sullo sfondo la società. Una società che proprio nel quinquennio preso in esame dal volume era attraversata da una serie di cambiamenti che ne avrebbero definitivamente mutato i connotati. Lo stesso PCI era pervaso in quei frangenti da uno sforzo finalizzato a leggere questi cambiamenti, caratterizzato da un grande interesse proprio verso quei meccanismi che molecolarmente stavano mutando il DNA del paese. Forse una maggiore attenzione ad aspetti di storia sociale e culturale avrebbe giovato a un volume di questa mole. Un volume che è comunque destinato a rimanere a lungo un punto di riferimento nel mondo degli studi sulla sinistra italiana. E un punto di riferimento per quanti combattono per una verità storica che si costruisce andando negli archivi e non nei salotti televisivi.

3 Cfr. CRAINZ, Guido, Il paese mancato. Dal miracolo economico agli anni Ottanta, Roma, Donzelli, 2003; ROSSANDA, Rossana, La ragazza del secolo scorso, Torino, Einaudi, 2005; MAGRI, Lucio, Il sarto di Ulm. Una possibile storia del PCI, Milano, Il Saggiatore, 2009. 


\section{* L’autore}

Marco Albeltaro, laureato in scienze politiche presso l'università di Torino, è dottorando in storia delle società contemporanee nel medesimo ateneo. Si occupa di storia delle idee politiche, di storia del movimento operaio e di storia del giornalismo. Oltre a numerosi saggi in riviste italiane e straniere ha curato L'assalto al cielo. Le ragioni del comunismo, oggi, Napoli, La Città del Sole, 2010 e ha pubblicato La parentesi antifascista. Giornali e giornalisti a Torino 19451948, Torino, SEB, 2011. Collabora al Dizionario biografico degli Italiani e all'Oxford Handbook of Communism. È redattore di «Historia Magistra. Rivista di storia critica».

URL: < http://studistorici.com/progett/autori/\#Albeltaro >

\section{Per citare questo articolo:}

ALBELTARO, Marco, «Recensione: Alexander, HÖBEL, /I PCl di Luigi Longo (1964-1969), Napoli, Edizioni Scientifiche Italiane, 2010, 626 pp.», Diacronie. Studi di Storia Contemporanea: Quando la classe operaia andava in paradiso, 13/2/2012,

URL:< http://www.studistorici.com/2012/02/13/albeltaro_numero_9/ >

\section{Diacronie Studi di Storia Contemporanea $\beta$ www.diacronie.it}

Risorsa digitale indipendente a carattere storiografico. Uscita trimestrale. redazione.diacronie@hotmail.it

Comitato di redazione: Marco Abram - Giampaolo Amodei - Jacopo Bassi - Luca Bufarale - Alessandro Cattunar - Alice De Rensis Barbara Galimberti - Deborah Paci - Fausto Pietrancosta - Martina Sanna - Matteo Tomasoni - Luca Zuccolo

Diritti: gli articoli di Diacronie. Studi di Storia Contemporanea sono pubblicati sotto licenza Creative Commons 2.5 . Possono essere riprodotti a patto di non modificarne i contenuti e di non usarli per fini commerciali. La citazione di estratti è comunque sempre autorizzata, nei limiti previsti dalla legge. 